\title{
Che spazio c'è per l'aferesi terapeutica in nefrologia? I trial di aferesi terapeutica in nefrologia
}

\author{
Ghil Busnach \\ Ospedale Niguarda Ca' Granda, Milano
}

\begin{abstract}
A REVIEW OF CONTROLLED TRIALS USING THERAPEUTIC APHERESIS IN NEPHROLOGY
Abstract. The role of therapeutic apheresis (TA) in renal diseases has long been under discussion, and although being the absolute numbers unchanged over the past 10-15 years, the indications have considerably changed. Many therapeutic changes have relied upon the demonstration, or lack of demonstration, of underlying mechanisms that would benefit from the use of plasma removal and/or treatment. In the literature there is a considerable criticism on the use of TA in nephrology, due to a lack of randomized controlled trials (RCT) and to the predominance of low-quality evidence. There are 4 different areas of interest for TA in nephrology, i.e. glomerular diseases, kidney transplantation, secondary nephropathies, and technical advances. To determine an improvement in the quality and quantity of evidence, the Clinicaltrials. gov database was searched at the end of 2012 for actively recruiting TA-RCT in nephrology.
\end{abstract}

Key words: Therapeutic apheresis, Renal disease, Clinical trials, Randomized controlled trials (RCT)

Conflict of interest: None.

Financial support: None.

Ricevuto: 14 Febbraio 2013; Accettato: 20 Marzo 2013

Le patologie renali rappresentano all'incirca il 16\% delle malattie trattate con aferesi terapeutica (AT). In questo ambito, nel 2010, il Canadian Apheresis Group indicava come patologia più frequentemente trattata la porpora trombotica trombocitopenica/sindrome emolitico-uremica (TTP/HUS), seguita dalle vasculiti associate a P e C-AN$\mathrm{CA}$, dal trapianto renale e, poi, da sindrome di Goodpasture, glomerulosclerosi focale recidiva dopo trapianto e crioglobulinemia, mentre risultava ridotto ai minimi termini l'impiego di AT per nefropatia da mieloma e LES (1). In gran parte per la relativa rarità di molte delle patologie renali trattate, ma anche a causa della frequente mancanza di criteri obiettivi a cui fare riferimento per valutare la modificazione indotta dall'aferesi, solo una piccola quota di AT viene svolta nell'ambito di studi clinici (2). Ancora inferiore è la quantità di trial controllati e randomizzati (RCT): il riferimento più attuale per conoscere la situazione degli studi pianificati, in corso o conclusi, è il sito $w w w$. ClinicalTrials.gov (3). Una ricerca condotta nell'Ottobre del 2012 usando come parola chiave "plasmaferesi" indicava 90 studi, dei quali 40 aperti alla data di ricerca. Inserendo come parole chiave "plasmaferesi" e "rene", la ricerca veniva ristretta a 36 studi, dei quali 17 aperti, come a indicare che quasi la metà degli studi clinici in campo aferetico può essere ricondotta a patologie renali. La progettazione e l'attuazione di un trial di aferesi in ambito nefrologico richiede un lungo lavoro di preparazione, in genere con lo scopo di pianificare un RCT, dal quale si presume di poter ottenere $\mathrm{i}$ dati più attendibili: uno studio randomizzato di fase 3, che preveda l'inserimento di un elevato numero di pazienti, si scontra, però, la maggior parte delle volte, con l'esiguità numerica delle patologie, a volte rare nel vero senso della parola e, comunque, sempre poco comuni: non a caso, una revisione di trial clinici osservava, nel 2010, che il numero di pubblicazioni di RCT rispetto alle pubblicazioni totali in ambito biomedico è aumentato tra il 1978 e il 2008 solo dallo $0.5 \%$ al $2.3 \%$, percentuale quest'ultima rimasta quasi invariata nell'ultimo decennio (4). Le piccole popolazioni e le malattie rare rappresentano una vera sfida alla progettazione di trial clinici. Se, infatti, una condizione patologica è rapidamente progressiva e un tentativo di cura mostra un miglioramento drammatico, qualsiasi successo (anche di un solo caso) può essere attribuito alla terapia (per esempio, la somministrazione di un fattore di coagulazione in caso di una carenza congenita). Viceversa, nel caso di una malattia con decorso cronico o con un andamento variabile oppure in cui lo scopo del trattamento non è la guarigione ma la 
palliazione, nessuna risposta singola al trattamento può essere attribuita alla terapia, come nel caso della somministrazione di IvIg nella sclerosi multipla. Da tutto questo consegue il fatto che l'impiego di RCT debba essere criticamente esaminato quando i risultati di una terapia sono immediatamente osservabili oppure quando, anche con il miglior trattamento possibile, morbilità e mortalità restano elevate, quando pazienti e medici appaiono riluttanti a scegliere o a essere scelti per un braccio di trattamento di controllo oppure quando i costi di un RCT appaiono proibitivi rispetto al possibile vantaggio. Da questo punto di vista, le situazioni fin qui esemplificate possono essere ricondotte per la grande maggioranza proprio a quei casi per i quali esistono indicazioni teoriche o pratiche per l'impiego di AT. Allo stato attuale, in ambito nefrologico, i trial clinici registrati da ClinicalTrials.gov, nel corso dei quali è previsto l'impiego di tecniche di aferesi terapeutica, possono essere suddivisi in 4 grandi categorie. Con la rara eccezione di studi che si propongono di valutare l'efficacia di singole tecniche aferetiche, come l'immunoadsorbimento o le rimozioni selettive o semiselettive, l'aferesi è parte di progetti terapeutici nel trattamento 1) di malattie glomerulari, 2) del trapianto renale, 3) di nefropatie secondarie e, in ultimo, 4) di altre condizioni (5). Tra le malattie glomerulari, i più importanti trial clinici che prevedono l'uso di aferesi sono rivolti al trattamento della glomerulosclerosi focale (GSF) e delle vasculiti associate alla presenza di anticorpi anti-citoplasma dei neutrofili (ANCA). Nel primo caso, lo studio "Permeability Factor in Focal Segmental Glomerulosclerosis", ClinicalTrials.gov NCT00007475, ha come scopo quello di identificare in pazienti affetti da GSF una popolazione con elevati livelli circolanti di una proteina permeabilizzante la membrana basale (FPF), di esaminarne i profili di espressione di RNA e di definire cinetiche di scomparsa e ricomparsa di FPF dopo immunosoppressori e nei casi di recidiva dopo trapianto, con AT. Il secondo trial in corso nell'ambito delle nefropatie prevalentemente glomerulari è "Plasma Exchange and Glucocorticoids for Treatment of Anti-Neutrophil Cytoplasm Antibody (ANCA)-Associated Vasculitis" (PEXIVAS), ClinicalTrials.gov NCT00987389, il cui proposito è di determinare se l'AT (così come la terapia immunosoppressiva) sia efficace nel ridurre la mortalità e l'uremia terminale irreversibile (ESRD) e anche se un regime di terapia steroidea ridotta sia efficace quanto quello standard. Lo studio, randomizzato, prevede, oltre a un'immunosoppressione con ciclofosfamide o rituximab, l'inserimento in uno di 4 bracci, steroide standard o a bassa dose e con o senza associazione con AT. Nell'ambito del trapianto renale, il ruolo dell'AT è oggi prevalentemente orientato alla prevenzione e al trattamento del rigetto umorale, da anticorpi anti-HLA donatore-specifici (DSA) e non, o alla preparazione del trapianto ABO incompatibile. Meno frequente, ma di importanza sostanziale, è il trattamento della recidiva della GSF dopo il trapianto. Il "Trial of Rituximab given pre-transplant to sensitized live donor kidney recipients" (RAPTURE), ClinicalTrials.gov NCT00371904, ha come obiettivo primario la valutazione dei rigetti umorali (AMR) a 1 anno dal trapianto, dopo un trattamento desensibilizzante che prevede l'impiego dell'AT, la cui efficacia è solo uno degli obiettivi secondari. Anche nello studio "Dosing regimen of Eculizumab added to conventional treatment in positi- ve crossmatch deceased donor kidney transplant", ClinicalTrials. gov NCT01106027, il trattamento con AT è di supporto all'obiettivo primario, che è la valutazione dell'efficacia e della sicurezza dell'impiego dell'Eculizumab nei trapianti renali da donatore cadavere con crossmatch positivo.

Nell'ambito della preparazione al trapianto $\mathrm{ABO}$ incompatibile, lo studio "ABO blood group antibody elimination by a combination of semiselective immunoadsorption therapy and membrane filtration", ClinicalTrials.gov NCT01698736, si propone di valutare l'efficacia di un solo trattamento con immunoadsorbimento semiselettivo associato o meno a una filtrazione su membrana di polisulfone nel ridurre IgM specifiche anti-ABO. Aspetti strettamente tecnici dell'AT sono presi in considerazione da due studi in corso: il primo, "Regional citrate anticoagulation in plasma exchange treatment", ClinicalTrials.gov NCT01370200, vuole valutare l'efficacia dell'AT nel rimuovere le IgG in corso di GSF e di sindrome di Guillain-Barré con l'impiego di due diverse concentrazioni di anticoagulante (citrato) nel circuito extracorporeo. Il secondo trial clinico, "The plasma large-volume exchange RCT" (PLEX-RCT), ClinicalTrials.gov NCT01433003, si propone di valutare, con un disegno randomizzato in aperto, di fase 3 , in un'ampia popolazione, l'efficacia dell'AT con lo scambio plasmatico di grandi volumi plasmatici in caso di TTP/HUS.

Alla TTP/HUS sono dedicati sia uno studio clinico che uno osservazionale: il trial "Rituximab in patients with relapsed or refractory TTP-HUS", ClinicalTrials.gov NCT00531089, ha come obiettivo la valutazione di efficacia e sicurezza del rituximab nell'indurre la remissione nei casi recidivi o refrattari e nell'abolire la necessità di AT. Lo studio osservazionale "Outbreak of hemolytic uremic syndrome linked to E. coli of serotype O104:H4 (SHU O104 CUB)", ClinicalTrials.gov NCT01406288, si propone di valutare la risposta alle terapie somministrate, compresa l'AT, sia come plasma exchange che come immunoadsorbimento, in occasione di un'epidemia di HUS osservata nel 2011 in Francia, dopo un banchetto, nella zona di Bordeaux.

Alla fine del 2012, risultavano completati gli arruolamenti degli studi "Trial of plasma exchange for acute renal failure at the onset of myeloma", "Study of the influence of plasma exchange on the pharmacokinetics of rituximab", "Anti-cytokine therapy for Vasculitis" (ACTIVE) e "Plasma exchange for autoimmune autonomic failure", mentre sono terminati e appena pubblicati o in corso di pubblicazione gli studi "Plasma exchange for renal vasculitis" (MEPEX) (6) e "Clinical outcome study of ARC1779 injection in patients with thrombotic microangiopathy".

Da ultimo, si ricorda il recente inserimento nel sito ClinicalTrials.gov di un progetto di ricerca italiano, "HELP-Apheresis in diabetic ischemic foot treatment" (HADIF), ClinicalTrials. gov NCT01518205, che si propone di verificare l'effetto di un'AT selettiva, come la LDL-aferesi, nel trattamento, in aggiunta alla terapia tradizionale, delle ulcere in pazienti con piede diabetico ischemico e vasculopatia periferica non rivascolarizzabile. Pur non essendo una tematica strettamente nefrologica, tuttavia, per la tipologia di pazienti a cui è rivolto, questo trial potrebbe fornire dati di grande interesse per la prevenzione e la cura della vasculopatia frequentemente osservata nei pazienti in trattamento sostitutivo dialitico. 


\section{Riassunto}

L'aferesi (AT) è una procedura terapeutica usata per trattare diverse patologie, mediante la rimozione in toto o in maniera selettiva del plasma o di alcune frazioni plasmatiche. In letteratura, l'uso dell'AT è spesso criticato per la qualità generalmente bassa dell'evidenza. Una revisione sistematica della letteratura permette di osservare un generale incremento delle pubblicazioni riguardanti l'AT, ma con un inadeguato incremento dei trial clinici randomizzati e controllati (RCT). In ambito nefrologico, i RCT in corso hanno sempre una numerosità piuttosto ridotta, mettendo, quindi, in discussione la loro reale capacità di determinare l'efficacia dell'AT come opzione terapeutica. È stata effettuata una ricerca sul database ClinicalTrials.gov che ha permesso di documentare gli studi RCT in corso nel 2012 in ambito nefrologico, che sono principalmente orientati al trattamento di 1) glomerulopatie, 2) trapianto renale e 3) nefropatie secondarie e alla valutazione di singole tecniche di AT.
Parole chiave: Aferesi terapeutica, Studi clinici, Malattie renali, Trial clinici, Trial randomizzati e controllati (RCT)

Dichiarazione di conflitto di interessi: L'Autore dichiara di non avere conflitto di interessi.

Contributi economici degli autori: L'Autore dichiara di non aver ricevuto sponsorizzazioni economiche per la preparazione dell'articolo.

Indirizzo degli Autori:

Dr. Ghil Busnach

Piazza Umanitaria 2

20122 Milano

omnibus@tiscali.it

\section{Bibliografia}

1. Clark WF. Plasma exchange for renal disease: evidence and use 2011. J Clin Apher 2012; 27: 112-6.

2. Winters JL. Randomized controlled trials in therapeutic apheresis. J Clin Apher 2013; 28 (1): 48-55.

3. http://www.clinicaltrials.gov/

4. Karlberg JPE, Speers MA (Eds). Reviewing Clinical Trials: A Guide for Ethics Committee. Karlberg JPE Publisher, Hong
Kong 2010.

5. Winters JL. Plasma exchange: concepts, mechanisms, and an overview of the American Society for Apheresis guidelines. Hematology Am Soc Hematol Educ Program 2012; 2012: 7-12.

6. Pepper RJ, Chanouzas D, Tarzi R, et al. Intravenous cyclophosphamide and plasmapheresis in dialysis-dependent ANCA-associated vasculitis. Clin J Am Soc Nephrol 2013; 8 (2): 219-24. 